\title{
A Study of Subracks
}

\author{
Samantha Driskill* \\ Department of Mathematics, Southwestern Oklahoma State University \\ *Student:driskills@student.swosu.edu \\ *Mentor: guy.biyogmam@swosu.edu
}

\begin{abstract}
Racks were introduced in 1959 by G. Wraith and J. Conway as a wreckage of groups, and have been used in various topics of mathematics. In this project, we aim to establish certain classical results known for subgroups on subracks.
\end{abstract}

\section{KEYWORDS}

Racks, Subracks, Quandle, Group, Centralizer, Dimension

\section{INTRODUCTION}

The theory of racks ${ }^{1}$ is strongly connected to the theory of conjugation in groups. Unlike groups, racks are non-associative algebraic structures. They appear in literature under several names such as automorphic set ${ }^{2}$, crystals $^{3}$ and left distributive left quasigroups ${ }^{4}$. Originally, racks were called "wracks" 5 , and were described as groups in which the group operation is discarded and only the concept of conjugation is left (see Example 2.2). A subrack of a rack is a subset that is a rack on its own. In this paper, we provide a characterization of subracks. As a result, we construct particular subracks of a given rack. In particular, we define the notions of the centralizer of an element in a rack and the center of a rack. We also introduce the notion of dimension on racks. In section 4 , we apply these notions in two examples.

\section{AJUR volume 12 | Issue 2 | January 2015}




\section{RACKS}

Let us recall $\left(\right.$ see $^{6}$ and $\left.^{7}\right)$ a few definitions.

- A Rack $(\mathrm{R}, \mathrm{o}, 1)$ is a set $\mathrm{R}$ with a binary operation $\circ$ and a specific element $1 \in R$ such that the following conditions are satisfied:

1. $x \circ(y \circ z)=(x \circ y) \circ(x \circ z)$

2. for each $x, y \in R$, there exits a unique $a \in R$ such that $x \circ a=y$

(1.2)

3. $1 \circ x=x$ and $x \circ 1=1$ for all $\mathrm{x} \in R$

In addition to these properties,

- A rack $R$ is involutive if it further satisfies $\quad x \circ(x \circ y)=y$ for all $x, y \in R$.

- A rack $R$ is abelian or trivial if it further satisfies $\quad x \circ y=y$ for all $x, y \in R$.

- A rack $R$ is a quandle if it further satisfies $\quad x \circ x=x$ for all $x \in R$.

- A Kei is an involutive quandle.

- The order of a rack $R$ (denoted $|R|)$ is the number of elements of $R$.

- Let $R, R^{\prime}$ be pointed racks. A function $\alpha: R \rightarrow R^{\prime}$ is said to be a homomorphism

of racks if $\quad \alpha\left(x_{1} \circ x_{2}\right)=\alpha\left(x_{1}\right) \circ \alpha\left(x_{2}\right)$ for all $x_{1}, x_{2} \in R$, and $\alpha(1)=1$.

\section{Example 2.1}

The following is a kei known as the Takasaki Kei $\mathfrak{T}=\{0,1,2,3,4,5\}$ of order 6 with operation $x \circ y=2 y-x \bmod 6$. Its Cayley table is given by

\begin{tabular}{|l|l|l|l|l|l|l|}
\hline$\circ$ & 0 & 1 & 2 & 3 & 4 & 5 \\
\hline 0 & 0 & 2 & 4 & 0 & 2 & 4 \\
\hline 1 & 5 & 1 & 3 & 5 & 1 & 3 \\
\hline 2 & 4 & 0 & 2 & 4 & 0 & 2 \\
\hline 3 & 3 & 5 & 1 & 3 & 5 & 1 \\
\hline 4 & 2 & 4 & 0 & 2 & 4 & 0 \\
\hline 5 & 1 & 3 & 5 & 1 & 3 & 5 \\
\hline
\end{tabular}

Example 2.2

A group $G$ endowed with the operation $\circ$ defined by $x \circ y=x y x^{-1}$ is a quandle (pointed by $1 \in G$ ) called the rack associated to the group $G$.

\section{AJUR volume 12 | Issue 2 | January 2015}




\section{Example 2.3}

Consider the dihedral group $D_{6}=\{\mathrm{e}, \mathrm{a}, \mathrm{b}, \mathrm{aba}, \mathrm{ab}, \mathrm{ba}\}$ of order 6 consisting of 3 reflections $\mathrm{a}, \mathrm{b}$ and $\mathrm{aba}$, and 3 rotations $\mathrm{e}, \mathrm{ab}$ and ba. Its Cayley table is provided below on the left. We construct the rack (call it $\mathfrak{D}_{6}$ ) associated to the dihedral group $D_{6}$. With the notations $\mathrm{c}:=\mathrm{aba}, \mathrm{d}:=\mathrm{ab}, \mathrm{f}:=\mathrm{ba}$ we provide below the Cayley tables:

\begin{tabular}{|c|c|c|c|c|c|c|}
\hline$\circ$ & $e$ & $a$ & $b$ & $c$ & $d$ & $f$ \\
\hline$e$ & $e$ & $a$ & $b$ & $c$ & $d$ & $f$ \\
\hline$a$ & $a$ & $e$ & $d$ & $f$ & $b$ & $c$ \\
\hline$b$ & $b$ & $f$ & $e$ & $d$ & $c$ & $a$ \\
\hline$c$ & $c$ & $d$ & $f$ & $e$ & $a$ & $b$ \\
\hline$d$ & $d$ & $c$ & $a$ & $b$ & $f$ & $e$ \\
\hline$f$ & $f$ & $b$ & $c$ & $a$ & $e$ & $d$ \\
\hline
\end{tabular}

The Dihedral group $D_{6}$

\begin{tabular}{|l|l|l|l|l|l|l|}
\hline$\circ$ & $e$ & $a$ & $b$ & $c$ & $d$ & $f$ \\
\hline$e$ & $e$ & $a$ & $b$ & $c$ & $d$ & $f$ \\
\hline$a$ & $e$ & $a$ & $c$ & $b$ & $f$ & $d$ \\
\hline$b$ & $e$ & $c$ & $b$ & $a$ & $f$ & $d$ \\
\hline$c$ & $e$ & $b$ & $a$ & $c$ & $f$ & $d$ \\
\hline$d$ & $e$ & $b$ & $c$ & $a$ & $d$ & $f$ \\
\hline$f$ & $e$ & $c$ & $a$ & $b$ & $d$ & $f$ \\
\hline
\end{tabular}

The Dihedral rack $\mathfrak{D}_{6}$

Note that $\mathfrak{D}_{6}$ is a non involutive quandle since $d \circ(d \circ c)=d \circ a=b \neq c$

\section{Proposition 2.4}

The rack associated to a cyclic group is abelian.

Proof. Let $G$ be a cyclic group and $R=(G$, o) the rack associated to the group $G$.

Let $x, y \in R$, we need to show that $x \circ y=y$

$$
\begin{aligned}
x \circ y & =a^{k} a^{l} a^{-k} \\
& =a^{k+l+-k} \\
& =a^{l} \\
& =y
\end{aligned}
$$

\section{SUBRACKS}

Recall that for a pointed $\operatorname{rack}(R,[-,-], 1)$, a non empty subset $S \subseteq R$ is called a subrack if $1 \in S$ and $(S, \circ, 1)$ has a pointed rack structure.

The following is a characterization of subracks.

\section{Theorem 3.1}

A non empty subset $S$ of a (pointed) $\operatorname{rack}(R, \circ, 1)$ is a subrack if and only if the following are satisfied:

$1.1 \in S$ If the rack is pointed

2. $S$ is closed under the binary operation o

3. For all $a, b \in R, a \circ b \in S$ and $a \in S$ implies $b \in S$

Proof. Assume that $S$ is a subrack of $R$. Then 1. and 2. are satisfied as $S \subseteq R$. To show 3., let $a, b \in R$ with $a \in S$ and $a \circ b \in S$. We need to show that $b \in S$. Set $c:=a \circ b$. Since $c \in S, a \in S$ and $S$ has a rack structure, there exists a unique $x \in S$ such that $a \circ x=c$. This means that $a \circ x=a \circ b$. So $x=b$ by uniqueness in (1.2). Thus, $b \in S$. Conversely, assume that 1., 2. and 3. are satisfied. To show that $S$ is a subrack, it remains to show (1.2). For this purpose, let $a, b \in S$. Since $S \subseteq R, a, b \in R$. So there exists a unique $x \in R$ such that $a \circ x=b$ by (1.2) for $R$. So $a \circ x \in S$. Now since $a \in S$, it follows by the hypothesis 3 . that $x \in S$. It is sufficient to take $c=x$ to complete the proof.

Now we prove several results on subracks using this characterization. 


\section{Proposition 3.2}

The set theoretical intersection of subracks of a rack $R$ is either empty or a subrack of $R$.

Proof. Let $\left\{S_{i}\right\}_{i \in I}$ be a family of subracks of $R$. If $R$ is pointed, then $1 \in \bigcap_{\mathrm{i} \in \mathrm{I}} S_{i}$ as $1 \in S_{i}$ for all $i \in I$. If $R$ is not pointed, then assume $\bigcap_{i \in \mathrm{I}} S_{i} \neq \emptyset$. Now for all $a, b \in \bigcap_{\mathrm{i} \in \mathrm{I}} S_{i}$ i.e. $a, b \in S_{i}$ for all $i \in I$, we have $a \circ b \in S_{i}$ for all $\mathrm{i} \in I$. Thus $a \circ b \in \bigcap_{\mathrm{i} \in \mathrm{I}} S_{i}$. Finally, if $a \circ b \in S_{i}$ and $a \in S_{i}$ for all $i \in I$, we have $b \in S_{i}$ for all $i \in \mathrm{I}$ by Theorem 3.1. Therefore $b \in \bigcap_{\mathrm{i} \in \mathrm{I}} S_{i}$. The result follows by Theorem 3.1.

\section{Definition 3.3}

Let $R$ be a rack and $M$ a subset of $R$. The subrack of $R$ generated by $M$ is the intersection of all subracks of $R$ containing $M$. It is therefore the smallest subrack of $R$ containing $M$. In particular, if $M$ is a subrack, then it generates itself.

\section{Definition 3.4}

A basis of a rack $R$ is a minimal subset of $R$ generating $R$. Its size is called the dimension of $R$.

\section{Example 3.5}

The Takasaki Kei $\mathfrak{I}$ and the Dihedral rack $\mathfrak{D}_{6}$ are both racks of order 6, but their dimension is 2 (see section 4).

The following theorem is analogue to Lagrange's theorem in group theory.

\section{Theorem 3.6}

For a rack $R$, the dimension of any subrack $S$ of $R$ is at most the dimension of $R$.

Proof. The proof is straightforward since a basis of $S$ is a subset of some basis of $R$ as $S \subseteq R$.

\section{Theorem 3.7}

Every (pointed) rack has an involutive subrack.

Proof. Let $K=\{a \in R \mid$ a $\circ(\mathrm{a} \circ x)=x \forall x \in R$. $\}$ Clearly, $K \subseteq R$. Also by (1.3),

$1 \circ(1 \circ x)=1 \circ x=x$ for all $x \in K$. So $1 \in K$. Let $a, b \in K$. Then by (1.1) successively,

$$
\begin{aligned}
(a \circ b) \circ((a \circ b) \circ x) & =((a \circ b) \circ(a \circ b)) \circ((a \circ b) \circ x) \\
& =a \circ(b \circ b) \circ(a \circ b) \circ(a \circ(a \circ x)) \\
& =a \circ(b \circ b) \circ a \circ(b \circ(a \circ x)) \\
& =a \circ(b \circ b) \circ(b \circ(a \circ x)) \\
& =a \circ b \circ(b \circ(a \circ x)) \\
& =a \circ(a \circ x) \\
& =x .
\end{aligned}
$$

So $a \circ b \in K$ and $K$ is closed under the operation o. Now Let $a, b \in R$ with $a \circ b \in K$ and $a \in K$. Then $b=a \circ(a \circ b) \in K$ since $K$ is closed under the operation $\circ$. The result follows by Theorem 3.1.

The following are analogue to the centralizer of an element and the center of a group.

\section{AJUR volume 12 | Issue 2 | January 2015}




\section{Proposition 3.8}

Let $R$ be a (pointed) rack and $u \in R$. Then

$$
C_{u}=\{x \in R \mid u \circ x=x\}
$$

is either empty or a subrack of $R$.

Proof. If $R$ is pointed, it is clear that $1 \in C_{u}$ as $u \circ 1=1$ by (1.3). If $R$ is not pointed, then assume $C_{u} \neq \emptyset$ and let $x, y \in C_{u}$.

$$
u \circ(x \circ y)=(u \circ x) \circ(u \circ y)=x \circ y \text {. }
$$

So $C_{u}$ is closed under the binary operation. Now Let $x \circ y \in C_{u}$ with $x \in C_{u}$

$$
x \circ(u \circ y)=(u \circ x) \circ(u \circ y)=u \circ(x \circ y)=x \circ y \text {. }
$$

By the uniqueness (1.2), $u \circ y=y$ and thus $y \in C_{u}$. The result follows by Theorem 3.1.

\section{Definition 3.9}

$C_{u}$ is called the centralizer of $u$.

\section{Corollary 3.10}

Let $R$ be a rack. Then

$$
Z(R)=\{x \in R \mid a \circ x=x, \forall a \in R\}
$$

is either empty or a subrack of $R$.

Proof. If $R$ is pointed, it is clear that $1 \in Z(R)$ as $u \circ 1=1$ for all $u \in R$. If $R$ is not pointed, then assume $Z(R) \neq \emptyset$. Then $Z(R)=\bigcap_{u \in R} C_{u}$ because

$$
\begin{aligned}
x \in \mathrm{Z}(\mathrm{R}) & \Leftrightarrow u \circ x=x \text { for all } u \in \mathrm{R} \\
& \Leftrightarrow x \in C_{u} \text { for all } u \in \mathrm{R} \\
& \Leftrightarrow x \in \bigcap_{u \in R} C_{u} .
\end{aligned}
$$

The result follows by Proposition 3.8 and Proposition 3.2.

\section{Definition 3.11}

$Z(R)$ is called the center of $R$.

\section{Remark 3.12}

The normalizer of a subrack $S$ of rack $R$ is the subrack itself. Indeed as in group theory, the normalizer of $S$ would be defined by

$$
\mathfrak{N}_{L}(S)=\{a \in R \mid u \circ a \in S, \forall u \in S\} .
$$

But it turns out that $S \subseteq \mathfrak{N}_{L}(S)$ since $S$ is closed under the rack operation o. Also for $a \in \mathfrak{N}_{L}(\mathrm{~S})$, $u \circ a \in S$ for all $u \in S$. This implies that $a \in S$ as $S$ is a subrack of $R$. Hence $\mathfrak{N}_{L}(S)=S$.

Proposition 3.13

Let $R, R^{\prime}$ be racks and $\varphi: R \rightarrow R^{\prime}$ a rack homomorphism. If $R^{\prime}$ is pointed, then the set

$$
K_{\varphi}=\{x \in R / \varphi(x)=1\}
$$

is a subrack of $R$.

Proof. If $R$ is pointed, $1 \in K_{\varphi}$ as $\varphi(1)=1$. Let $u, v \in K_{\varphi}$ i.e. $\varphi(\mathrm{u})=1$ and $\varphi(v)=1$. Then $\varphi(a \circ b)=$ $\varphi(a) \circ \varphi(b)=1 \circ 1=1$. So $a \circ b \in K_{\varphi}$. Now let $a, b \in \mathrm{R}$ with $a \circ b \in K_{\varphi}$ and $a \in K_{\varphi}$. Then $\varphi(b)=1$ $\circ \varphi(b)=\varphi(a) \circ \varphi(b)=\varphi(a \circ b)=1$. This implies $b \in K_{\varphi}$. The result follows by Theorem 3.1. 
Definition 3.14

$K_{\varphi}$ is called the kernel of the rack homomorphism $\varphi$.

Proposition 3.15

Let $R, R^{\prime}$ be (pointed) racks with $R^{\prime}$ involutive. Let $\varphi: R \rightarrow R^{\prime}$ be a homomorphism. Then the image of $\varphi$

is a subrack of $R^{\prime}$.

$$
\operatorname{Im} \varphi:=\{\varphi(x), x \in R\}
$$

Proof. If $R$ and $R^{\prime}$ are pointed, the $1 \in \operatorname{Im} \varphi$ because $1=\varphi(1)$. Let $y_{1}, y_{2} \in \operatorname{Im} \varphi$ i.e. $y_{1}=\varphi\left(x_{1}\right)$ for some $x_{1} \in R$ and $y_{2}=\varphi\left(x_{2}\right)$ for some $x_{2} \in R$. Then $y_{1} \circ y_{2}=\varphi\left(x_{1}\right) \circ \varphi\left(x_{2}\right)=\varphi\left(x_{1} \circ x_{2}\right) \in \operatorname{Im} \varphi$. So $\operatorname{Im} \varphi$ is closed under the operation o of $R^{\prime}$. Now let $y_{1}, y_{2} \in R$ with $y_{1} \circ y_{2} \in \operatorname{Im} \varphi$ and $y_{1} \in \operatorname{Im} \varphi$. We need to show that $y_{2} \in \operatorname{Im} \varphi$. Indeed $y_{1}=\varphi\left(x_{1}\right)$ and $y_{1} \circ y_{2}=\varphi(z)$ for some $x_{1}, z \in R$. Since $R^{\prime}$ is involutive, we have

$$
y_{2}=y_{1} \circ\left(y_{1} \circ y_{2}\right)=y_{1} \circ \varphi(z)=\varphi(x 1) \circ \varphi(z)=\varphi\left(x_{1} \circ z\right) \in \operatorname{Im} \varphi .
$$

The result follows by Theorem 3.1.

\section{EXAMPLES}

\section{Example 4.1}

The following are the Cayley tables of the subracks of the Takasaki Kei $\mathfrak{I}$ of Example 2.1.

$S_{0}=\{0\}$
$S_{1}=\{1\}$

$S_{6}$

\begin{tabular}{|l|l|l|}
\hline$\circ$ & 0 & 3 \\
\hline 0 & 0 & 0 \\
\hline 3 & 3 & 3 \\
\hline
\end{tabular}

$S_{7}$

\begin{tabular}{|c|c|c|c|}
\hline \multirow{3}{*}{$S_{8}$} & o & 2 & 5 \\
\hline & 2 & 2 & 2 \\
\hline & 2 & 5 & 5 \\
\hline
\end{tabular}

\begin{tabular}{|c|c|c|}
\hline$\circ$ & 1 & 4 \\
\hline 1 & 1 & 1 \\
\hline 4 & 4 & 4 \\
\hline
\end{tabular}

$S_{2}=\{2\}$

$S_{3}=\{3\}$

$S_{4}=\{4\}$

$S_{5}=\{5\}$
$S_{9}$

\begin{tabular}{|l|l|l|l|}
\hline$\circ$ & 0 & 2 & 4 \\
\hline 0 & 0 & 4 & 2 \\
\hline 2 & 4 & 2 & 0 \\
\hline 4 & 2 & 0 & 4 \\
\hline
\end{tabular}

$S_{10}$

\begin{tabular}{|l|l|l|l|}
\hline$\circ$ & 1 & 3 & 5 \\
\hline 1 & 1 & 5 & 3 \\
\hline 3 & 5 & 3 & 1 \\
\hline 5 & 3 & 1 & 5 \\
\hline
\end{tabular}

$S_{11}=\mathfrak{I}$ 


\section{Remarks 4.2}

1. $\mathfrak{T}$ has exactly 11 subracks.

2. All the subracks of $\mathfrak{I}$ are also Takasaki Keis of lower order.

3. The dimension of $S_{9}$ is 2 as $S_{9}$ has basis either of the subsets $\{0,2\},\{0,4\}$ or $\{2,4\}$.

4. The dimension of $S_{10}$ is 2 as $S_{10}$ has basis either of the subsets $\{1,3\},\{1,5\}$ or $\{3,5\}$.

5. The dimension of $\mathfrak{I}$ is 2 as $\mathfrak{I}$ has basis either of the subsets $\{s, r\}$, with $s \in\{0,2,4\}$ and $\{1,3,5\}$.

6. The center of $\mathfrak{T}$ is empty.

7. The centralizers are as follows: $C_{0}=\{0\}, C_{1}=\{1\}, C_{2}=\{2\}, C_{3}=\{3\}, C_{4}=\{4\}, C_{5}=\{5\}$

\section{Example 4.3}

The following are the Cayley tables of the subracks of the Dihedral rack $\mathfrak{D}_{6}$ (Example 2.3).
$S_{0}=\{e\}$
$S_{2}=\{b\}$
$S_{4}=\{d\}$
$S_{1}=\{a\}$
$S_{3}=\{c\}$
$S_{5}=\{f\}$

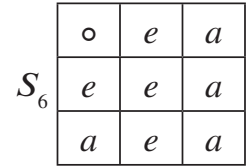

\begin{tabular}{|c|c|c|c|c|}
\hline \multicolumn{1}{|c|}{$S_{12}$} & \multicolumn{1}{|c|}{$a$} & $b$ & $c$ \\
\cline { 2 - 5 } & $a$ & $a$ & $c$ & $b$ \\
\cline { 2 - 5 } & $b$ & $c$ & $b$ & $a$ \\
\hline$c$ & $b$ & $a$ & $c$ \\
\hline
\end{tabular}

$S_{7}$

\begin{tabular}{|l|l|l|}
\hline$\circ$ & $e$ & $b$ \\
\hline$e$ & $e$ & $b$ \\
\hline$b$ & $e$ & $b$ \\
\hline
\end{tabular}

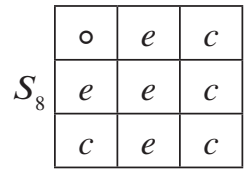

\begin{tabular}{|c|c|c|c|}
\hline \multirow{3}{*}{$S_{9}$} & 0 & $e$ & $d$ \\
\hline & $e$ & $e$ & $d$ \\
\hline & $d$ & $e$ & $d$ \\
\hline
\end{tabular}

$S_{10}$\begin{tabular}{|l|l|l|}
\hline$\circ$ & $e$ & $f$ \\
\cline { 2 - 4 }$e$ & $e$ & $f$ \\
\hline$f$ & $e$ & $f$ \\
\hline
\end{tabular}

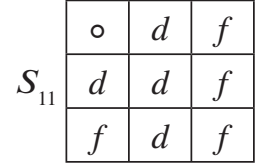

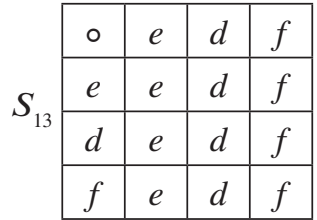

$S_{1}$

\begin{tabular}{|l|l|l|l|l|}
\hline$\circ$ & $e$ & $a$ & $b$ & $c$ \\
\hline$e$ & $e$ & $a$ & $b$ & $c$ \\
\hline$a$ & $e$ & $a$ & $c$ & $b$ \\
\hline$b$ & $e$ & $c$ & $b$ & $a$ \\
\hline$c$ & $e$ & $b$ & $a$ & $c$ \\
\hline
\end{tabular}

$S_{15}$

\begin{tabular}{|c|c|c|c|c|c|}
\hline$\circ$ & $a$ & $b$ & $c$ & $d$ & $f$ \\
\hline$a$ & $a$ & $c$ & $b$ & $f$ & $d$ \\
\hline$b$ & $c$ & $b$ & $a$ & $f$ & $d$ \\
\hline$c$ & $b$ & $a$ & $c$ & $f$ & $d$ \\
\hline$d$ & $b$ & $c$ & $a$ & $d$ & $f$ \\
\hline$f$ & $c$ & $a$ & $b$ & $d$ & $f$ \\
\hline
\end{tabular}

$S_{16}=\mathfrak{D}_{6}$ 


\section{Remarks 4.4}

1. The center of $\mathfrak{D}_{6}$ is $S_{0}$.

2. The rack $\mathfrak{D}_{6}$ has exactly 17 subracks.

3. Every subrack of $\mathfrak{D}_{6}$ is involutive except $S_{15}$ and $\mathfrak{D}_{6}$.

4. The centralizers are as follows: $C_{e}=\mathfrak{D}_{6}, C_{a}=S_{6}, C_{b}=S_{7}, C_{c}=S_{8}, \mathrm{C}_{d}=S_{13}, \mathrm{C}_{f}=S_{13}$.

5. The subrack $S_{12}$ is the Dihedral rack $\mathfrak{D}_{3}$ of order 3 .

6. The only non abelian subracks of $\mathfrak{D}_{6}$ are $S_{12}, S_{14}, S_{15}$ and $\mathfrak{D}_{6}$.

7. The dimension of $S_{14}$ is 3 as $S_{14}$ has basis the subset $\{e, a, b\}$.

8. The dimension of $S_{15}$ is 2 as $S_{15}$ has basis the subset $\{a, d\}$.

9. The dimension of $\mathfrak{D}_{6}$ is 3 as $\mathfrak{D}_{6}$ has basis either of the subsets $\{e, s, r\}$ with $s \in\{a, b, c\}$ and $r \in\{d, f\}$.

\section{Remark 4.5}

In group theory, Lagrange's theorem states that for a group $G$, the order of any subgroup $H$ of $G$ divides the order of $G$. This statement is not true for racks. As a counterexample, $S_{14}$ is a subrack of the Dihedral rack $\mathfrak{D}_{6}$ above, but $\left|S_{14}\right|=4$ does not divide $6=\left|\mathfrak{D}_{6}\right|$.

\section{Remark 4.6}

The set theoretical union of two subracks is not a subrack. As a counterexample, $S_{6}$ and $S_{13}$ are subracks of the The Dihedral rack $\mathfrak{D}_{6}$ above, but $S_{6} \cup S_{13}$ is not a subrack of $\mathfrak{D}_{6}$.

\section{Remark 4.7}

One can check that the map $\varphi: \mathfrak{T} \rightarrow \mathfrak{D}_{6}$ defined by:
$\varphi(0)=a$,
$\varphi(2)=c$,
$\varphi(4)=b$,
$\varphi(1)=b$,
$\varphi(3)=a$,
$\varphi(5)=c$.

is a rack homomorphism.

Its image is $\operatorname{Im} \varphi=S_{12}$.

\section{AJUR volume 12 | Issue 2 | January 2015}




\section{ACKNOWLEDGEMENTS}

The author gives special thanks to the Southwestern Oklahoma State University mathematics department for the support and to Dr. Guy Biyogmam for the supervision of this undergraduate project.

\section{REFERENCES}

[1] Fenn, R., and Rourke, C. (1992) Racks and links in codimension two, J. Knot Theory Ramifications $1,343-406$.

[2] Brieskorn, E. (1988) Automorphic sets and braids and singularities, Contemp. Math. 78, 45-115.

[3] Kauffman, L. H. (1991) Knots and Physics, World Scientific, 1st ed., Singapore.

[4] Stanovský, D. (2004) Left distributive left quasigroups, PhD Thesis, Charles University in Prague, retrieved 23 September, 2014, from http://www.karlin.mff.cuni.cz/ stanovsk/math/disert.pdf.

[5] Conway, J., and Wraith, G. (1958), Unpublished correspondence.

[6] Andruskiewitsch, N., and Graña, M. (2003) From racks to pointed Hopf algebras, Adv. Math. 178(2), $177-243$.

[7] Bozhüyük, M. E. (1993) Topics in Knot theory, Kluwer Academic Publishers, Dordrecht, The Netherlands.

\section{ABOUT THE STUDENT AUTHOR}

Samantha Driskill worked on this project during her senior year (2012-2013) at Southwestern Oklahoma State University. She graduated in the spring of 2014 with two Bachelor of Science degrees (Mathematics and Mathematics Education). She was the president of the Mathematics club and a recipient of multiple awards in the math department. Samantha is currently a full-time teacher at Del city high school in Oklahoma.

\section{PRESS SUMMARY}

The theory of racks is strongly connected to the theory of conjugation in groups. Roughly, racks are described as groups in which the group operation is discarded and only the concept of conjugation is left. In this paper, we investigate and introduce on racks some notions well-developed in groups, and apply those notions in a few examples. 